\title{
Basic and Translational Understandings of Microbial Recognition by Toll-Like Receptors in the Intestine
}

\author{
Sang Hoon Rhee \\ Division of Digestive Diseases, David Geffen School of Medicine, University of California Los Angeles, Los Angeles, CA, USA
}

Microbial recognition by multicellular organisms is initially accomplished by a group of pattern recognition receptors which are specialized to recognize microbe-associated molecular patterns (MAMPs) such as lipopolysaccharide, bacterial lipoprotein, CpG DNA motif, double strand RNA and flagellin. Toll-like receptors (TLRs) are the representative pattern recognition receptors, and microbial recognition by TLRs elicits innate and inflammatory responses. Ten TLR family members have been presently identified in human genome, and numerous studies discovered that intracellular responses from MAMPs-TLR engagements are mediated by a participation of at least 4 immediate adaptor molecules such as myeloid differentiation primary response gene-88 (MyD88), MyD88 adaptor-like (Mal) (also known as Toll/L-1 receptor domain-containing adaptor protein [TIRAP]), Toll/L-1 receptor domain-containing adaptor-inducing interferon- $\beta$ (TRIF) and TRIF-related adaptor molecule (TRAM) leading to activate transcription factors including nuclear factor $\mathrm{KB}$, activator protein-1 and interferon-regulatory factors. Given that large amounts of commensal microbiota constantly reside in the intestinal lumen, enteric microbial recognition by TLRs at the intestinal epithelium provides a critical impact on regulating intestinal homeostasis. Indeed, aberrant TLR4 and TLR5 activations are etiologically associated with the development and progress of intestinal inflammatory diseases including inflammatory bowel disease and necrotizing enterocolitis. In this review article, we present the molecular mechanism by which TLRs elicit intracellular signal transduction, and summarize the physiological relevance of TLRs related to the gastrointestinal tract.

(J Neurogastroenterol Motil 2011;17:28-34)

Key Words

Flagellin; Immunity, innate; Toll-like receptor

\section{Introduction}

In the human gastrointestinal tract, there are up to $1 \times 10^{12}$ bacterial cells per gram of colonic content, and gut microflora is constituted by 400-1,000 species of bacteria. ${ }^{1,2}$ Balanced in- testinal microflora plays a direct impact on maintaining the gut homeostasis such as regulating bowel motility, modulating intestinal pain, processing nutritional factors and inducing immune responses. ${ }^{3-5}$ These commensal microbes are recognized by pattern recognition receptors (PRRs), which in turn regulate mucosal innate immunity and inflammatory responses. Among

Received: December 1, 2010 Revised: December 22, 2010 Accepted: December 28, 2010

(c) This is an Open Access article distributed under the terms of the Creative Commons Attribution Non-Commercial License (http://creativecommons. org/licenses/by-nc/3.0) which permits unrestricted non-commercial use, distribution, and reproduction in any medium, provided the original work is properly cited.

*Correspondence: Sang Hoon Rhee, PhD

Division of Digestive Diseases, David Geffen School of Medicine, UCLA, MRL1240, 675 Charles E. Young Drive South, Los Angeles, CA 90095, USA

Tel: +1-310-794-5148, Fax: +1-310-825-3542, E-mail: shrhee@mednet.ucla.edu

Financial support: This work was supported in part by "Young Clinical Scientist Award" (S.H.R.) from "Flight Attendant Medical Research Institute, Conflicts of interest: None. Inc" and by the National Institutes of Health, DK079015 (S.H.R.). 
PRRs, Toll-like receptors (TLRs) are the first-discovered and the most well characterized member of PRRs, and detect microbe-associated molecular patterns (MAMPs) present in a wide range of microorganisms. ${ }^{6}$ Here we present the molecular and cellular mechanism of TLR-mediated responses and their clinical implication in the gastrointestinal tract. Given the fact that the gastrointestinal tract of mammalian hosts harbors vast range of microorganism and the intestinal epithelial cells constituting the intestinal epithelium constantly contact with enteric microflora at a front-line of host-microbial communication, this review will be mainly focused on microbial recognition in the intestinal epithelial cells.

\section{Physiological Effects of Intestinal Microflora on Gut Homeostasis}

Imbalanced microbiota in the gut is known to be one of the etiological reasons provoking mucosal inflammation such as inflammatory bowel disease (IBD). ${ }^{2,7}$ Moreover, bacterial flagellin, one of many microbial products, was recently described as a dominant antigen in IBD patients. ${ }^{8} \mathrm{We}$ and others demonstrated that immune responses triggered by microbial products are associated with intestinal inflammation. ${ }^{4,8-11}$ Moreover, the gut microbiota is an important environmental factor that affects intestinal motility, resulting in irritable bowel syndrome (IBS). ${ }^{12,13}$ For instance, intestinal microflora elicits both structural and functional responses in the sensory and motor apparatus of the gut by increasing the factors mediating neuronal responses in enteric neurons. ${ }^{13,14}$ Bifidobacterium bifidum and Lactobacillus acidophilus are able to promote motility, while Escherichia species can inhibit this effect. ${ }^{3}$ In contrast, several studies suggest inhibitory effects of enteric bacteria on regulating gut motility. ${ }^{15,16}$ Moreover, microbial factors such as short-chain fatty acids, bacterial endotoxin or chemotactic peptides (eg, formyl-methionyl-leucyl-phenylalanine) released from intestinal bacteria are able to stimulate the enteric nervous system in order to affect gut motility. ${ }^{13,17,18}$ In this context, therefore, disruption of gut microflora is poised to alter gut immunity and motility associated with aberrant bowel physiology.

\section{Microbial Recognition by Pattern Recognition Receptors}

Since living organisms are in a continuous contact with microbial milieu including commensal and pathogenic microbes, the host-microbial communication such as recognizing the presence of microbes and responding to microbial factors is one of the critical measures for innate immunity and commensal relationship between host and microflora. The host-microbial interaction is accomplished by a molecular sensor of microbial recognition in which microbial sensory system specifically perceives a characteristic molecular pattern (eg, lipopolysaccharide [LPS], flagellin, CpG DNA, single or double strand RNA) from various microorganisms. Since the microbial sensory system specifically recognizes and binds to a microbial pattern molecule, the sensory system is regarded as PRRs. ${ }^{6}$ PRRs recognize microbes in multicellular organisms, resulting in activating cellular responses against the microorganism: activation of innate and adaptive immunity and inflammatory responses. ${ }^{6}$

\section{Toll-Like Receptors}

TLRs are the type I transmembrane protein, which is composed of leucine rich repeat domain at the extracellular region, transmembrane domain and cytoplasmic Toll/IL-1 receptor (TIR) domain. ${ }^{19,20}$ While leucine rich repeat domain is specialized to recognize a specific microbial ligand, TIR domain is responsible for mediating the intracellular signaling generated by the engagement between TLRs and MAMPs. ${ }^{21}$ In human genome, so far ten TLRs have been identified: TLR1, TLR2, TLR3, TLR4, TLR5, TLR6, TLR7, TLR8, TLR9 and TLR10. ${ }^{22}$ TLRs specifically recognize and bind to MAMPs: for instance, TLR4 and TLR5 are the receptor for LPS and flagellin, respectively (Table). Based on the cellular localization,

Table. Representative Toll-Like Receptors and Their Specific Ligands

\begin{tabular}{|c|c|}
\hline TLRs & MAMPs \\
\hline \multirow[t]{4}{*}{ TLR2 } & Bacterial lipoproteins (eg, Pam3Cys and MALP-2) \\
\hline & Lipoteichoic acid (LTA) \\
\hline & Peptidoglycan (PGN) \\
\hline & Zymosan \\
\hline TLR3 & double strand RNA (dsRNA) (eg, Poly [I:C]) \\
\hline TLR4 & Lipopolysaccharide (LPS) \\
\hline TLR5 & Flagellin \\
\hline TLR7, TLR8 & Single strand RNA (ssRNA), \\
\hline & Small anti-viral compounds \\
\hline TLR9 & Unmethylated CpG DNA \\
\hline
\end{tabular}

TLRs, toll-like receptors; MAMPs, microbe-associated molecular patterns; Pam3Cys, a synthetic ligand of TLR2; MALP-2, Macrophage-activating lipopeptide-2. 
TLRs are categorized into 2 groups: plasma membrane TLRs including TLR1, TLR2, TLR4, TLR5 and TLR6 residing in the plasma membrane and endosomal TLRs including TLR3, TLR7, TLR8 and TLR9 present at intracellular endosomes. ${ }^{22}$

TLRs make a homo- or hetero-complex (eg, TLR1/TLR2 and TLR2/TLR6) to expedite microbial sensing, and recruit an array of immediate adaptor molecules to its cytoplasmic TIR domain upon a ligand recognition. ${ }^{21,23}$ Intensive studies identified at least 4 adaptor molecules such as myeloid differentiation primary response gene-88 (MyD88), Mal/TIRAP (Mal is also called TIR domain-containing adaptor protein [TIRAP]), TIR domain-containing adaptor-inducing interferon- $\beta$ (TRIF) and TRIF-related adaptor molecule (TRAM) interacting with TLRs in response to ligand stimulation. ${ }^{21,24}$ These adaptor molecules harbor a TIR domain responsible for the interaction with the cytoplasmic TIR domain of TLRs. When TLR recognizes a microbial ligand, it recruits a single or a combination of adaptor molecule(s) to its cytoplasmic TIR domain. ${ }^{24}$ Subsequently, these adaptor molecules associate with IL-1 receptor-associated kinases (IRAKs) to mediate the signaling to a member of TNF receptor-associated factor (TRAF) family (eg, TRAF6). Thereby, TLR-induced signaling leads to activation of inhibitory $\mathrm{K} B$ kinases (IKKs) and mitogen-activate protein kinases (MAPKs) to activate transcription factors such as nuclear factor $\kappa \mathrm{B}(\mathrm{NF}-\kappa \mathrm{B})$, activator protein-1 (AP-1) and interferon-regulatory factors (IRFs), followed by inducing a pleiotropic gene expression involved in immune and inflammatory responses. ${ }^{22,24,25}$

\section{Toll-Like Receptors in the Gastrointestinal Tract and Their Clinical Relevance}

In order to appreciate the impact of enteric microbiota on gut physiology, thus, it is very critical to understand how intestinal epithelial cells recognize enteric microbiota and what are the consequences resulted from microbial recognition by the intestinal epithelial cells. Having found that TLRs are the representative microbial recognition sensor at the cell membrane as well as inside a cell, TLRs at intestinal epithelial cells should be a key sensory system in recognizing enteric microbes, which in turn alters the intracellular signaling within the gut epithelial cells and leads to activating innate and inflammatory responses.

Intriguingly, most of intestinal epithelial cell lines are potently responsive to flagellin which is a specific ligand for TLR5, ${ }^{26,27}$ and hyporesponsive to TLR2 and TLR4 (eg, Pam3Cys and LPS, respectively) activation. ${ }^{28,29}$ Despite of the hypo-responsiveness of TLR4 in various intestinal epithelial cells, uncontrolled TLR4 activation is clearly responsible for necrotizing enterocolitis which is a well known pediatric intestinal inflammatory disease. ${ }^{30}$ Preterm infants show a higher expression level of TLR4 at the intestine than normal term infants, which renders preterm infants highly susceptible to the intestinal inflammation due to TLR4 activation by enteric microbes obtained after delivery. ${ }^{30,31}$ Moreover, baby formula has been known to upregulate TLR4 expression in the intestine, which is probably associated with one of the reason for pediatric intestinal inflammation. ${ }^{32}$ In addition, TLR4 deficiency makes the mouse susceptible to dextran sulfate sodium-induced colitis, while feeding LPS to normal mice provides protective effects against dextran sulfate sodium-induced colitis. ${ }^{33}$ These studies suggest that TLR4 activation by LPS in the intestine may provide beneficial effects such as promoting epithelial cell proliferation resulting in enhanced wound healing at the intestinal epithelium. Therefore, LPS/TLR4 engagement is believed to be one of the most important factors governing the intestinal physiology.

Given the fact that most intestinal epithelial cells are strongly responsive to flagellin, ${ }^{26,27}$ TLR5 should be considered as an important microbial sensor providing a significant impact on intestinal epithelial cells which are not only critical for maintaining the intact epithelial barrier function, but highly associated with the activation of lamina propria cells at the submucosa. Indeed, aberrant TLR5 activation appears to be etiologically associated with the development and progress of IBD. ${ }^{4,8}$ Intriguingly, TLR 5 seems to be asymmetrically localized at the basolateral side of epithelial mucosa. ${ }^{4,34}$ Therefore, impaired barrier function of the epithelium escalates responsiveness to flagellin, which may be responsible for exacerbated intestinal inflammation. ${ }^{35}$ Despite many studies suggesting that flagellin/TLR5 engagement promotes intestinal inflammation, ${ }^{4,8,11,36-39}$ some studies imply that flagellin protects mucosal epithelium against mucosal insults (eg, radiation). ${ }^{40,41}$ Interestingly, Gewirtz's group reported that $10 \%$ of TLR5-knockout (KO) mice exhibited spontaneous colitis in a TLR4-dependent manner, ${ }^{42,43}$ indicating that TLR5 may protect from mucosal inflammation. However, they also observed that such spontaneous colitis in TLR5-KO mice was not observed in "rederived" TLR5-KO mice. ${ }^{43}$ Moreover, the other TLR5-KO mice independently generated by Flavell's group have normal growth, size, fertility and lifespan, and show no obvious abnormalities. ${ }^{44}$ Thus, further studies are needed to determine whether TLR 5 promotes or protects from colitis. In addition to the involvement of TLR5 in mucosal inflammation, our recent 
study showed that TLR5 activation could elicit potent anti-tumor immunity against colon cancer by promoting neutrophil infiltration to tumor legions. ${ }^{45}$

TLR3 specifically recognizing double-strand RNA from viruses elicits potent innate immune responses by inducing IFN- $\beta$ production against rotavirus which is one of well known enteric viruses. ${ }^{46}$ TLR9 can also be activated in intestinal epithelial cells transfected with CpG DNA motifs. ${ }^{47}$ Since bacterial DNA harbors a large amount of CpG DNA motifs, TLR9 should be activated by $\mathrm{CpG}$ DNA released from enteric bacteria in the intestine.

\section{Toll-Like Receptor-Induced Signaling in the Intestinal Epithelial Cells}

Although TLR3-, TLR4-, TLR5- and TLR9-induced responses have been demonstrated in the intestinal epithelial cells, ${ }^{26,27,48-50}$ among various TLRs are TLR4 and TLR5 which have been intensively investigated as it pertains to the gastrointestinal diseases. In this review, therefore, TLR-activated molecular mechanism will be primarily addressed on TLR4- and TLR5-mediated responses. TLR4 makes a heteromeric receptor complex with CD14 and myeloid differentiation-2 at the plasma membrane in order to expedite LPS recognition. ${ }^{21,23} \mathrm{Mal} /$ TIRAP adaptor molecule is then recruited to the plasma membrane and binds with the cytoplasmic TIR domain of TLR4, followed by recruiting MyD88 adaptor molecule. Recruiting Mal/TIRAP and MyD88 to TLR4 at the plasma membrane leads to activating serine-threonine kinase IRAKs which subsequently interact with TRAF6. TRAF6 is a cytosolic adaptor molecule mediating IKKs and MAPKs, which subsequently activates transcription factors $\mathrm{NF}-\kappa \mathrm{B}$ and $\mathrm{AP}-1$, respectively. ${ }^{51,52}$ Meanwhile, LPS binding to the heteromeric complex of TLR4 also causes additional adaptor recruitment of TRAM. The complex is then endocytosed to endosome in which TRIF adaptor molecule is recruited to TLR4 complex. Like Mal/TIRAP for MyD88, TRAM adaptor appears to play a bridging role for TRIF to be recruited to TLR4. ${ }^{51,52}$ Once TRIF is associated with TLR4, it primarily induces transcription factor IRF-3 activation mediating IFN- $\beta$ production. Collectively, the combinations of Mal/TIRAP-MyD88 and TRAM-TRIF adaptors are involved in TLR4-induced signaling pathways, in which the former is called the MyD88-dependent pathway mostly associated with NF- $\kappa \mathrm{B}$ and AP-1 activation, and the latter is the MyD88-independent pathway mainly linked to IRF-3 activation for anti-viral defense by producing IFN- $\beta$ (Figure).

TLR5 residing in the plasma membrane has been known to utilize only MyD88 adaptor, resulting in activating NF- $\kappa \mathrm{B}$ and AP-1. ${ }^{53,54}$ Our recent study, however, showed that TRIF is recruited to TLR5 at least in the intestinal epithelial cells to regulate NF- $\kappa \mathrm{B}$ and AP-1 activation, while TRIF associated with TLR5 does not appear to elicit IRF-3 activation for IFN- $\beta$ production. ${ }^{55}$ Our study also determined that TRAM is not re-

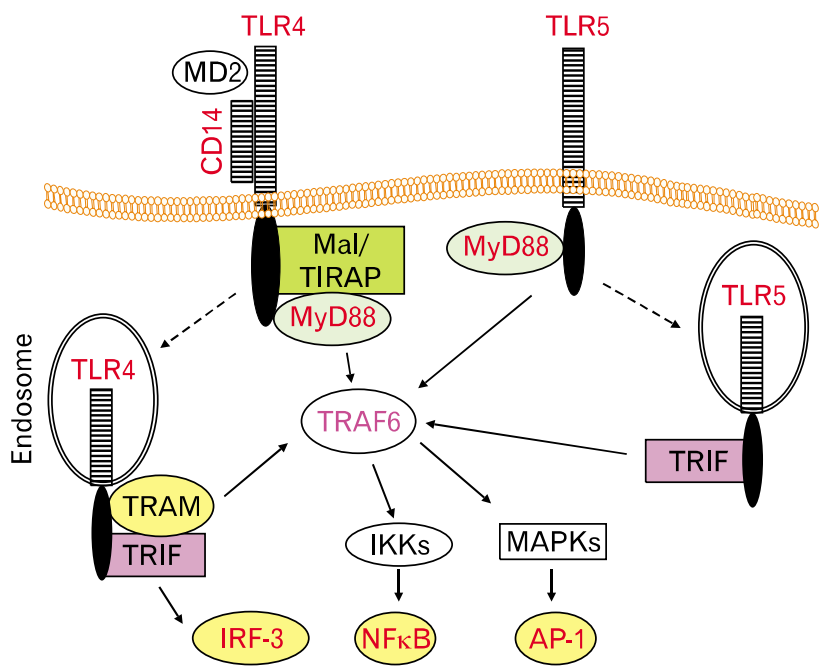

Figure. Simplified comparison of toll-like receptor (TLR) 4- and TLR5-induced signaling pathways. Lipopolysaccharide (LPS) engagement induces the heteromeric complex formation of TLR4, CD14 and myeloid differentiation protein-2 at the plasma membrane. Then, Mal/TIRAP (Mal is also called Toll/IL-1 Receptor [TIR] domaincontaining adaptor protein [TIRAP]) adaptor is first recruited to the cytoplasmic TIR domain of TLR4, followed by recruiting myeloid differentiation primary response gene-88 (MYD88) adaptor. Next, it activates serine-threonine kinase IL-1 receptor-associated kinases (not shown) to recruit TNF receptor-associated factor (TRAF) 6 adaptor. TRAF6 relays the signaling to activate inhibitory $\mathrm{K} B$ kinases and mitogen-activate protein kinases (MAPKs), resulting in nuclear factor $\kappa \mathrm{B}(\mathrm{NF}-\kappa \mathrm{B})$ and activator protein-1 (AP-1) transcription factor activation. In parallel, LPS-stimulated TLR4 complex also recruits TIR domain-containing adaptor-inducing interferon- $\beta$ (TRIF)-related adaptor molecule adaptor to its cytoplasmic region, and the complex is internalized to endosome where the complex interacts with TRIF adaptor in order to activate interferon-regulatory factor-3 transcription factor activation. In contrast, TLR5 activation by flagellin results in recruiting MyD88 adaptor at the plasma membrane, followed by activating $\mathrm{NF}-\kappa \mathrm{B}$ and $\mathrm{AP}-1$ transcription factor. In similar, TLR5 also utilizes TRIF at the endosome to activate NF- $\kappa \mathrm{B}$ and AP-1 transcription factor. IKKs, inhibitory $\mathrm{K} B$ kinases; IRF-3, interferon-regulatory factor-3; MD2, myeloid differentiation protein-2; TRAM, TRIFrelated adaptor molecule. 
cruited to TLR5 upon flagellin stimulation. ${ }^{55}$ Upon flagellin stimulation, TLR5 seems to be internalized to endosome in which TRIF can be attached to TLR5. ${ }^{56}$ Based on these studies, TLR5 exploits MyD88 and TRIF as adaptors in order to induce $\mathrm{NF}-\kappa \mathrm{B}$ and AP-1 activation regulating pleiotropic expression of proinflammatory cytokine gene in response to flagellin. In contrast to TLR4 and TLR5, TLR9 is known to use only MyD88 for NF- $\kappa \mathrm{B}$ and AP-1 activation, where TLR3 exclusively utilizes TRIF in order to induce IFN- $\beta$ expression. ${ }^{21}$

\section{Microbial Recognition by Non-pattern Recognition Receptors in Enterochromaffin Cells}

Like intestinal epithelial cells, enterochromaffin (EC) cells residing in the mucosa are in a continuous contact with gut microflora, and communicate with microflora in the gut, and a wide variety of receptor expression (eg, 5-hydroxytryptamine [5-HT] receptors, pituitary adenylate cyclase-activating polypeptide receptor, $\alpha$-adrenergic receptor, $\beta$-adrenergic receptor, cholinergic receptor, corticotropin-releasing hormone receptor, $\gamma$-aminobutyric acid receptor and TLRs) have been suggested in these cells. ${ }^{57,58}$ EC cells act as mucosal sensory transducers and secrete 5-HT in response to various physiological and pathological luminal stimuli. Engagements of these receptors by microbial factors or bacterial toxins seem to trigger a delicate mechanism for the production of 5-HT in EC cells, ${ }^{59-61}$ thereby modulate the gut motility. Unfortunately, microbial factors stimulating receptors on EC cells and the molecular and cellular mechanisms have yet to be studied.

Nonetheless, although microbial recognition is primarily mediated by PRRs like TLRs, some bacterial products are able to interact with its own specific receptor which is not a member of PRRs. For instances, cholera toxin, a secretory enterotoxin from Vibrio cholerae, binds to its receptor GM1 ganglioside in EC cells. A recent study showed that odorants present in the luminal environment stimulate olfactory receptors expressed in EC cells, resulting in 5-HT production. ${ }^{62}$

\section{Conclusion}

TLRs specifically recognize the presence of microorganisms, and there are large numbers of microbes in the gut. Thus, the gastrointestinal tract is the primary organ in which microbial recognition by TLRs should be essential to maintain the home- ostasis. Indeed, impaired microbial recognition by either altered gut microflora or genetic defects of PRRs turned out to be responsible for certain type of gastrointestinal disease. ${ }^{2,7}$ Moreover, emerging evidence suggests that microbial recognition in the gut elicits potent innate immunity against pathogenic microbes or tumors. Therefore, studying TLRs in the gastrointestinal tract is now attracting more research interest than any other research areas. While numerous studies have been focused on determining the complex intracellular signaling pathways and the involvement of signaling molecules in TLR-dependent responses, studies on clinical relevance of TLRs in the gastrointestinal tract still remain unclear. Thus, during the next decade, translational and clinical researches on this area will be aimed to determine the disease relevance of TLRs or TLR-dependent signaling pathways, which in turn pave the way to develop new therapeutic approaches of the gastrointestinal disease including IBD and IBS.

\section{References}

1. Rhee SH, Pothoulakis C, Mayer EA. Principles and clinical implications of the brain-gut-enteric microbiota axis. Nat Rev Gastroenterol Hepatol 2009;6:306-314.

2. Garrett WS, Gallini CA, Yatsunenko T, et al. Enterobacteriaceae act in concert with the gut microbiota to induce spontaneous and maternally transmitted colitis. Cell Host Microbe 2010;8:292-300.

3. Husebye E, Hellström PM, Sundler F, Chen J, Midtvedt T. Influence of microbial species on small intestinal myoelectric activity and transit in germ-free rats. Am J Physiol Gastrointest Liver Physiol 2001;280:G368-G380.

4. Rhee SH, Im E, Riegler M, Kokkotou E, O'brien M, Pothoulakis C. Pathophysiological role of Toll-like receptor 5 engagement by bacterial flagellin in colonic inflammation. Proc Natl Acad Sci USA 2005;102:13610-13615.

5. Ait-Belgnaoui A, Han W, Lamine F. Lactobacillus farciminis treatment suppresses stress induced visceral hypersensitivity: a possible action through interaction with epithelial cell cytoskeleton contraction. Gut 2006;55:1090-1094.

6. Palm NW, Medzhitov R. Pattern recognition receptors and control of adaptive immunity. Immunol Rev 2009;227:221-233.

7. Willing BP, Dicksved J, Halfvarson J, et al. A pyrosequencing study in twins shows that gastrointestinal microbial profiles vary with inflammatory bowel disease phenotypes. Gastroenterology 2010;139: 1844-1854.e1.

8. Lodes MJ, Cong Y, Elson CO, et al. Bacterial flagellin is a dominant antigen in Crohn disease. J Clin Invest 2004;113:1296-1306.

9. Leaphart CL, Cavallo J, Gribar SC, et al. A critical role for TLR4 in the pathogenesis of necrotizing enterocolitis by modulating intestinal injury and repair. J Immunol 2007;179:4808-4820.

10. Chan KL, Wong KF, Luk JM. Role of LPS/CD14/TLR4-mediated inflammation in necrotizing enterocolitis: pathogenesis and therapeutic implications. World J Gastroenterol 2009;15:4745-4752. 
11. Carvalho FA, Barnich N, Sauvanet P, Darcha C, Gelot A, Darfeuille-Michaud A. Crohn's disease-associated Escherichia coli LF82 aggravates colitis in injured mouse colon via signaling by flagellin. Inflamm Bowel Dis 2008;14:1051-1060.

12. Rodríguez-Fandiño O, Hernández-Ruiz J, Schmulson M. From cytokines to toll-like receptors and beyond - current knowledge and future research needs in irritable bowel syndrome. J Neurogastroenterol Motil 2010;16:363-373.

13. Barbara G, Stanghellini V, Brandi G, et al. Interactions between commensal bacteria and gut sensorimotor function in health and disease. Am J Gastroenterol 2005;100:2560-2568.

14. Hooper LV, Wong MH, Thelin A, Hansson L, Falk PG, Gordon JI. Molecular analysis of commensal host-microbial relationships in the intestine. Science 2001;291:881-884.

15. Guarino MP, Altomare A, Stasi E, et al. Effect of acute mucosal exposure to Lactobacillus rhamnosus GG on human colonic smooth muscle cells. J Clin Gastroenterol 2008;42(suppl 3 Pt 2):S185-S190.

16. Wang B, Mao YK, Diorio C, et al. Luminal administration ex vivo of a live Lactobacillus species moderates mouse jejunal motility within minutes. FASEB J 2010;24:4078-4088.

17. Dass NB, John AK, Bassil AK, et al. The relationship between the effects of short-chain fatty acids on intestinal motility in vitro and GPR43 receptor activation. Neurogastroenterol Motil 2007;19:6674.

18. Malbert $\mathrm{CH}$. The ileocolonic sphincter. Neurogastroenterol Motil 2005;17(suppl 1):41-49.

19. Medzhitov R, Preston-Hurlburt P, Janeway CA Jr. A human homologue of the Drosophila Toll protein signals activation of adaptive immunity. Nature 1997;388:394-397.

20. Rhee SH, Hwang D. Murine TOLL-like receptor 4 confers lipopolysaccharide responsiveness as determined by activation of $\mathrm{NF}$ kappa $\mathrm{B}$ and expression of the inducible cyclooxygenase. J Biol Chem 2000;275:34035-34040.

21. Akashi-Takamura S, Miyake K. TLR accessory molecules. Curr Opin Immunol 2008;20:420-425.

22. Medzhitov R. Recognition of microorganisms and activation of the immune response. Nature 2007;449:819-826.

23. Triantafilou M, Gamper FG, Haston RM, et al. Membrane sorting of toll-like receptor (TLR)-2/6 and TLR2/1 heterodimers at the cell surface determines heterotypic associations with CD36 and intracellular targeting. J Biol Chem 2006;281:31002-31011.

24. Kenny EF, O'Neill LA. Signalling adaptors used by Toll-like receptors: an update. Cytokine 2008;43:342-349.

25. Kawai T, Akira S. Toll-like receptor and RIG-I-like receptor signaling. Ann N Y Acad Sci 2008;1143:1-20.

26. Rhee SH, Jones BW, Toshchakov V, Vogel SN, Fenton MJ. Toll-like receptors 2 and 4 activate STAT1 serine phosphorylation by distinct mechanisms in macrophages. J Biol Chem 2003;278:2250622512.

27. Honko AN, Mizel SB. Effects of flagellin on innate and adaptive immunity. Immunol Res 2005;33:83-101.

28. Abreu MT, Arnold ET, Thomas LS, et al. TLR4 and MD-2 expression is regulated by immune-mediated signals in human intestinal epithelial cells. J Biol Chem 2002;277:20431-20437.

29. Abreu MT, Vora P, Faure E, Thomas LS, Arnold ET, Arditi M. Decreased expression of Toll-like receptor-4 and MD-2 correlates with intestinal epithelial cell protection against dysregulated proinflammatory gene expression in response to bacterial lipopolysaccharide. J Immunol 2001;167:1609-1616.

30. Gribar SC, Sodhi CP, Richardson WM, et al. Reciprocal expression and signaling of TLR4 and TLR9 in the pathogenesis and treatment of necrotizing enterocolitis. J Immunol 2009;182:636-646.

31. Wolfs TG, Derikx JP, Hodin CM, et al. Localization of the lipopolysaccharide recognition complex in the human healthy and inflamed premature and adult gut. Inflamm Bowel Dis 2010;16:68-75.

32. LeBouder E, Rey-Nores JE, Raby AC, et al. Modulation of neonatal microbial recognition: TLR-mediated innate immune responses are specifically and differentially modulated by human milk. J Immunol 2006; 176:3742-3752.

33. Rakoff-Nahoum S, Paglino J, Eslami-Varzaneh F, Edberg S, Medzhitov R. Recognition of commensal microflora by toll-like receptors is required for intestinal homeostasis. Cell 2004;118:229- 241.

34. Gewirtz AT, Navas TA, Lyons S, Godowski PJ, Madara JL. Cutting edge: bacterial flagellin activates basolaterally expressed TLR 5 to induce epithelial proinflammatory gene expression. J Immunol 2001; 167:1882-1885.

35. Hollander D. Crohn's disease - a permeability disorder of the tight junction? Gut 1988;29:1621-1624.

36. Ivison SM, Himmel ME, Hardenberg G, et al. TLR5 is not required for flagellin-mediated exacerbation of DSS colitis. Inflamm Bowel Dis 2010;16:401-409.

37. Targan SR, Landers CJ, Yang H, et al. Antibodies to CBir1 flagellin define a unique response that is associated independently with complicated Crohn's disease. Gastroenterology 2005;128:2020-2028.

38. Sitaraman SV, Klapproth JM, Moore DA 3rd, et al. Elevated flagellin-specific immunoglobulins in Crohn's disease. Am J Physiol Gastrointest Liver Physiol 2005;288:G403-G406.

39. Gewirtz AT, Vijay-Kumar M, Brant SR, Duerr RH, Nicolae DL, Cho JH. Dominant-negative TLR5 polymorphism reduces adaptive immune response to flagellin and negatively associates with Crohn's disease. Am J Physiol Gastrointest Liver Physiol 2006;290:G1157G1163.

40. Burdelya LG, Krivokrysenko VI, Tallant TC, et al. An agonist of toll-like receptor 5 has radioprotective activity in mouse and primate models. Science 2008;320:226-230.

41. Vijay-Kumar M, Aitken JD, Sanders CJ, et al. Flagellin treatment protects against chemicals, bacteria, viruses, and radiation. J Immunol 2008; 180:8280-8285.

42. Vijay-Kumar M, Sanders CJ, Taylor RT, et al. Deletion of TLR5 results in spontaneous colitis in mice. J Clin Invest 2007;117:39093921.

43. Vijay-Kumar M, Aitken JD, Carvalho FA, et al. Metabolic syndrome and altered gut microbiota in mice lacking Toll-like receptor 5. Science 2010;328:228-231.

44. Feuillet V, Medjane S, Mondor I, et al. Involvement of Toll-like receptor 5 in the recognition of flagellated bacteria. Proc Natl Acad Sci USA 2006;103:12487-12492.

45. Rhee SH, Im E, Pothoulakis C. Toll-like receptor 5 engagement modulates tumor development and growth in a mouse xenograft model of human colon cancer. Gastroenterology 2008;135:518-528.

46. Xu J, Yang Y, Sun J, et al. Expression of Toll-like receptors and their association with cytokine responses in peripheral blood mononuclear 
cells of children with acute rotavirus diarrhoea. Clin Exp Immunol 2006;144:376-381

47. Sánchez-Muñoz F, Fonseca-Camarillo GC, Villeda-Ramirez MA, et al. TLR9 mRNA expression is upregulated in patients with active ulcerative colitis. Inflamm Bowel Dis 2010;16:1267-1268.

48. Furrie E, Macfarlane S, Thomson G. Toll-like receptors-2, -3 and -4 expression patterns on human colon and their regulation by $\mathrm{mu}-$ cosal-associated bacteria. Immunology 2005;115:565-574.

49. Suzuki M, Hisamatsu T, Podolsky DK. Gamma interferon augments the intracellular pathway for lipopolysaccharide (LPS) recognition in human intestinal epithelial cells through coordinated upregulation of LPS uptake and expression of the intracellular Toll-like receptor 4-MD-2 complex. Infect Immun 2003;71:3503-3511.

50. Ewaschuk JB, Backer JL, Churchill TA, Obermeier F, Krause DO, Madsen KL. Surface expression of Toll-like receptor 9 is upregulated on intestinal epithelial cells in response to pathogenic bacterial DNA. Infect Immun 2007;75:2572-2579.

51. Kagan JC, Medzhitov R. Phosphoinositide-mediated adaptor recruitment controls Toll-like receptor signaling. Cell 2006;125:943955.

52. Kagan JC, Su T, Horng T, Chow A, Akira S, Medzhitov R. TRAM couples endocytosis of Toll-like receptor 4 to the induction of interferon-beta. Nat Immunol 2008;9:361-368.

53. Rhee SH, Keates AC, Moyer MP, Pothoulakis C. MEK is a key modulator for TLR5-induced interleukin-8 and MIP3alpha gene expression in non-transformed human colonic epithelial cells. J Biol Chem 2004;279:25179-25188.
54. Rhee SH, Kim H, Moyer MP, Pothoulakis C. Role of MyD88 in phosphatidylinositol 3-kinase activation by flagellin/toll-like receptor 5 engagement in colonic epithelial cells. J Biol Chem 2006;281: 18560-18568.

55. Choi YJ, Im E, Chung HK, Pothoulakis C, Rhee SH. TRIF mediates Toll-like receptor 5-induced signaling in intestinal epithelial cells. J Biol Chem 2010;285:37570-37578.

56. Choi YJ, Im E, Pothoulakis C, Rhee SH. TRIF modulates TLR5dependent responses by inducing proteolytic degradation of TLR5. J Biol Chem 2010;285:21382-21390.

57. Hansen MB, Witte AB. The role of serotonin in intestinal luminal sensing and secretion. Acta Physiol (Oxf) 2008;193:311-323.

58. Bogunovic M, Davé SH, Tilstra JS. Enteroendocrine cells express functional Toll-like receptors. Am J Physiol Gastrointest Liver Physiol 2007;292:G1770-G1783.

59. Grider JR, Kuemmerle JF, Jin JG. 5-HT released by mucosal stimuli initiates peristalsis by activating $5-\mathrm{HT} 4 / 5-\mathrm{HT} 1 \mathrm{p}$ receptors on sensory CGRP neurons. Am J Physiol 1996;270(5 Pt 1):G778-G782.

60. Peregrin AT, Ahlman H, Jodal M, Lundgren O. Involvement of serotonin and calcium channels in the intestinal fluid secretion evoked by bile salt and cholera toxin. Br J Pharmacol 1999;127:887-894.

61. Raybould HE. Visceral perception: sensory transduction in visceral afferents and nutrients. Gut 2002;51(suppl 1):111-i14.

62. Braun T, Voland P, Kunz L, Prinz C, Gratzl M. Enterochromaffin cells of the human gut: sensors for spices and odorants. Gastroenterology 2007;132:1890-1901. 